\title{
A Space-Time Multilevel Method For Molecular Dynamics Simulations
}

\author{
Haim Waisman and Jacob Fish \\ Department of Civil, Mechanical and Aerospace Engineering, \\ Rensselaer Polytechnic Institute, \\ Troy, NY 12180-3590
}

\begin{abstract}
We present a novel multiscale approach for molecular dynamics simulations. It is aimed at bridging discrete scales with either coarse grained discrete or continuum scales. The method consists of the waveform relaxation scheme aimed at capturing the high frequency response of atomistic vibrations and a coarse scale solution in space and time aimed at resolving smooth features (in both space and time domains) of the discrete medium. The method is implicit in space and time, possesses superior stability properties and consequently enables larger time steps governed by accuracy considerations of coarse scale quantities of interest. Performance studies on polymer melts have shown significant speed-up compared to the classical explicit methods, in particular on parallel machines.
\end{abstract}

Key words: Waveform relaxation, Space-Time Multilevel, Multigrid, Molecular Dynamics integration, Hessian matrix, Aggregation

\section{Introduction}

Molecular dynamics (MD) simulations provide the methodology for detailed fine scale modeling on the molecular level. MD can be viewed as a process by which one generates atomic trajectories of a system of particles by direct

* Corresponding author: fishj@rpi.edu (Jacob Fish)

1 The financial supports of National Science Foundation under grants CMS0310596, 0303902, 0408359 and Sandia National Laboratory under contract DEACD4-94AL85000 are gratefully acknowledged. In particular, the authors wish to thank Dr. Ray Tuminaro from Sandia National Laboratory for his constructive suggestions 
numerical integration of Newton's equations of motion with the appropriate initial and boundary conditions [1]. The system can be formulated either by dynamic equilibrium consideration or by means of variational principle and is written as

$$
\left\{\begin{array}{l}
M \ddot{d}=F^{\text {int }}(d)+F^{\text {ext }} \\
d(0)=d_{0} \\
\dot{d}(0)=v_{0}
\end{array}\right.
$$

where $d$ is a vector of atom positions, $M$ is the mass matrix, $F^{e x t}$ is a vector of external forces, and $F^{i n t}=-\nabla \Phi(d)$ is the internal force vector defined as the gradient of the potential energy.

Current MD algorithms severely restrict the modeling efforts to relatively small systems and/or short time intervals. The algorithmic challenges facing MD simulations stem from the difficulty of designing methods which are insensitive in terms of the integration time step to rapid fluctuations in chemical bonds and short ranged forces. Most widely used algorithms in MD are explicit methods, such as Verlet [2,3] and Gear's predictor-corrector methods [4]. A severe limitation in the ability of the explicit methods to propagate numerical trajectories stems from a wide range of time scales spanning many orders of magnitude. For instance, in polymer chains, bond-stretching vibrations are the fastest atomic motions in a molecule, typically on the order of femtoseconds, whereas the relaxation of polymers in the form of segmental motions or terminal relaxations of chains is spanning the time scales in the range between $10^{-2} s$ to $10^{4} s[5]$. The maximum time step is limited by the smallest oscillation period that can be found in the simulated system. This time step is necessary to maintain stability of explicit numerical integration schemes [6].

Considerable efforts have been devoted to increasing the critical time step. Many insightful observations on the numerical properties of the algorithms were reported, and numerous improvements have been proposed. One common approach is to constrain bond lengths using either SHAKE or RATTLE algorithms [7]. By this approach, bonds are constrained to have fixed length. Another commonly used approach in MD simulations is to employ variable time step using multiple-time-step (MTS) methods [8, 9, 10]. Nevertheless, the increases in the integration time step have been quite modest so far [11]. The temporal gap can be partially circumvented by parallel algorithms. However, due to inherently sequential nature of time integration schemes, parallelism has been exploited in the spatial domain only, while computations in the time domain remain primarily sequential. One promising research avenue is to develop integration algorithms, which are parallelizable in time domain. A different (or complimentary) approach is to exploit implicit integration. Implicit methods require solution of coupled nonlinear equations at every time step, which is both expensive and difficult to implement. Nevertheless, they reduce stability restrictions and enable much larger time step, typically governed by accuracy considerations $[12,13]$. In general, accuracy means how close is the solution of atomic trajectories obtained by the numerical integration to the 
true trajectories. We refer to this type of definition as the accuracy in local quantities. Molecular systems, however, are highly nonlinear and exhibit sensitive dependence on perturbations. Moreover, the initial conditions are usually assigned randomly. Thus, accurate approximation of local trajectories on meaningful time intervals is neither obtainable nor desired. This suggests analyzing the accuracy with respect to the global quantities of interest, such as temperature, energy, diffusion and others.

In the present manuscript we present a novel multiscale approach based on the space-time variational multilevel principle for molecular systems characterized by diverse spatial and temporal scales. Our approach is implicit in both space and time domains and uses typically 10 to 50 times larger time steps than explicit methods selected based on the accuracy of global fields of interest. The space-time character of the method provides an improved stability without directly solving the space-time problem. The multiscale nature of the system is exploited in two ways: First, it substantially reduces the cost of multilevel iterations (see Section 2.1), and secondly, it is parallelizable in the time domain by exploiting the waveform relaxation (WR) scheme in the smoothing process (see Section 2.2).

To this end we note that multigrid methods have been applied to MD simulations in the context of long range electrostatic force computations $[14,15,16]$. Another interesting multigrid-like approach has been employed for equilibration of large atomistic systems with linear scaling [17]. It is based on a separate treatment of long and short range components of force-fields. The rapidly varying part is handled by conventional optimization methods while slowly varying components have been captured by the elasticity theory.

In the paper we utilize multilevel methods for direct time integration of MD equations in addition to effectively solving the spatial problem. To our knowledge, the temporal aspect of the proposed method is original and has not been studied elsewhere. The paper is organized as follows. In Section 3 we develop the variational space-time multilevel method suitable for MD simulations, which exploits the multiscale nature of the problem. It consists of the WR scheme aimed at capturing the response atomistic vibrations and the coarse scale solution in space and time intended to resolve the slow features of the discrete medium. The coarse grid problem is derived from Hamilton's principle on the subspace of coarse scale functions. In Section 4 we show that for linear case the method reduces to the sequential multilevel method [18]. Performance studies are conducted in Section 5. 


\section{Motivation}

\subsection{Multigrid as a multiscale solver}

The motivation for use of multigrid ideas for multiscale problems was given in $[19,20]$. To convey the basic ideas, consider a continuum one-dimensional two-scale elliptic problem for the scalar field $\mathrm{u}$

$$
\left\{\begin{array}{l}
\frac{d}{d x}\left(k(x) \frac{d u}{d x}\right)+b(x)=0 \\
u(0)=u(L)=0
\end{array}\right.
$$

on the interval $x \in[0, L]$ with oscillatory periodic piecewise constant coefficients $k_{1}$ and $k_{2}$ and 0.5 volume fraction. That is every unit cell is combined of a constant stiffness $k_{1}$ and $k_{2}$ such that each occupies half of the cell size. In many applications of interest and in particular those described at the atomistic scale, $k_{1} \gg k_{2}$ yields highly heterogenous systems. Equation (2) is discretized using finite elements or finite difference leading to a linear system $K u=f$. Analyzing the eigenvalues of $K$ reveals the following. The eigenvalues are clustered at the two ends of the spectrum on the right half plane. More importantly, the eigenvalues clustered close to zero are identical to those obtained by the problem with homogenized coefficients $[19,20]$. This character of the spectrum suggests a computational strategy based on the philosophy of multigrid methods. In such a multigrid strategy the smoother is designed to capture the higher frequency response of the fine scale model represented by a linear combination of the highest eigenmodes. The auxiliary coarse model is then engineered to effectively capture the remaining lower frequency response of the fine scale problem. For a periodic heterogeneous medium, such an auxiliary coarse model coincides with the boundary value problem with homogenized coefficients as evidenced by the identical eigenvalues. The resulting multiscale prolongation operator is given by

$$
Q=Q^{c}+Q^{f}
$$

where $Q^{c}$ and $Q^{f}$ are the classical (smooth) prolongation and the fine scale correction obtained from the discretization of the influence functions, respectively. The rate of convergence of the multigrid process for the two-scale problem given by Eq. (2) is governed by [19, 20]

$$
\left\|e_{i+1}\right\|=\frac{q}{4-q}\left\|e_{i}\right\|
$$

where $\left\|e_{i+1}\right\|$ is the norm of the error at iteration $i+1$ and

$$
q=\frac{2 \sqrt{k_{1} k_{2}}}{\left(k_{1}+k_{2}\right)} .
$$


Note that for $k_{1}=k_{2}$ the media is homogeneous, and one recovers the classical multigrid rate of $\frac{1}{3}$ [21]. However, for example if either $\frac{k_{1}}{k_{2}}$ or $\frac{k_{2}}{k_{1}}$ is 100 , then the convergence factor reduces to $\frac{1}{5}$ and the two-scale process converges in five iterations up to the tolerance of $10^{-5}$. In this manuscript we devise a multigridlike strategy for efficient solution of MD equations. We view the integration of Newton's equations of motion as problem on a space-time grid. This observation suggests a unique space-time multilevel approach that exploits multiple spatial and temporal scales of a molecular system.

\subsection{The waveform relaxation scheme}

One of the major barriers for the efficient solution of large scale MD equations is the sequential character of time integration methods. The waveform relaxation (WR) method is an iterative solution of space-time problems that offers parallelization $[22,23]$. Due to its implicit nature, WR provides superior stability and large time steps compared to explicit methods.

In the WR algorithm, the space-time domain is partitioned in space into smaller subsystems, thereafter to be referred to as the spatial blocks or simply blocks. Each block is then integrated over the temporal grid. The spatial blocks can be integrated independently on different processors. The temporal grid is divided into intervals called windows. Windows are used to accelerate convergence and to reduce storage. At every time step an algebraic system of equations is solved on a spatial grid. See Figure 1 for definitions. Information

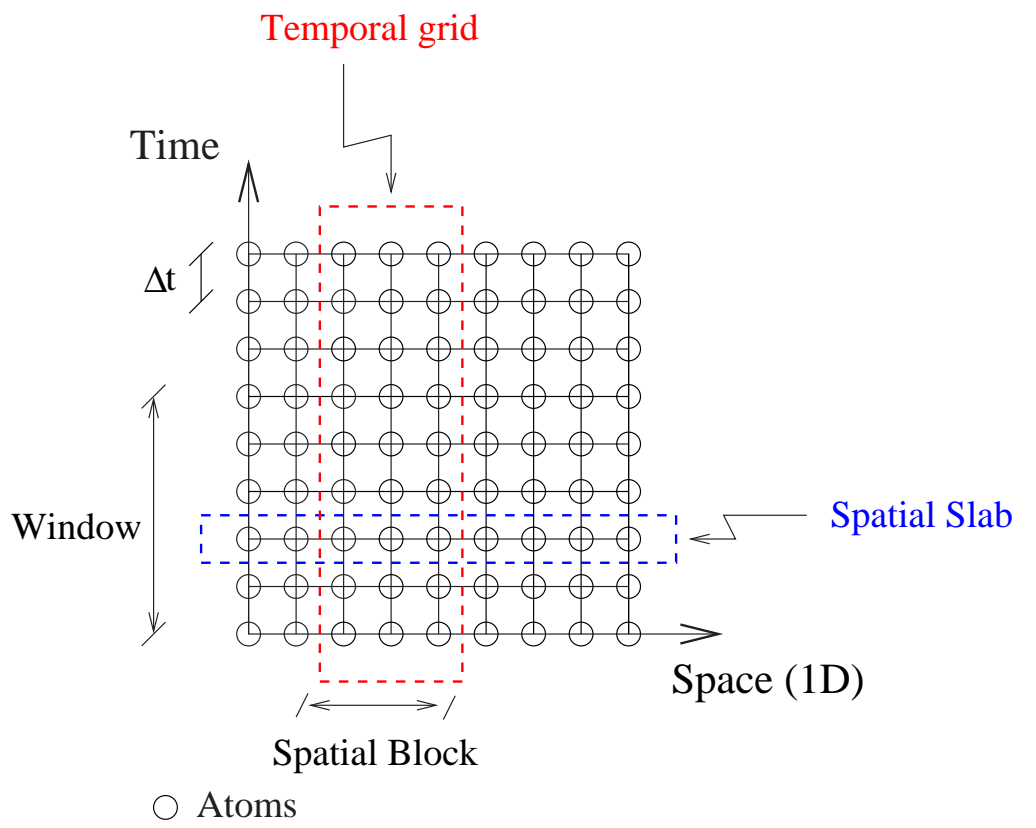

Fig. 1. Slabs, windows and blocks in the waveform relaxation method

transfer between different windows takes place once the integration over the 
corresponding windows is completed. The main advantage of the method is that it permits simultaneous integration of spatial blocks in each window and its ability for unstructured integration. For instance, spatial blocks involving stiff connections will require smaller time steps than those having more compliant connections analogous to the multiple time step (MTS) techniques [11]. The WR method has been primarily used in electrical network simulations and parabolic initial value problems. Limited studies have been conducted for hyperbolic [24] and second order [25] systems. In the remaining of this section we give some technical details of the WR method focusing on nonlinear systems. For highly nonlinear systems, such as those arising from MD simulations, two versions of WR method are common. The first is a direct extension of the linear WR formulations, so called Waveform Relaxation Newton (WRN) method [26]. In the MD context, the basic idea is to solve the following nonlinear scalar equations

$$
\left\{\begin{array}{l}
m_{i} \ddot{d}_{i}^{\nu+1}=F^{i n t}\left(d_{1}^{\nu}, \ldots, d_{i-1}^{\nu}, d_{i}^{\nu+1}, d_{i+1}^{\nu}, \ldots, d_{N}^{\nu}\right) \\
d_{i}^{\nu+1}(0)=d_{0_{i}} \\
\dot{d}_{i}^{\nu+1}(0)=v_{0_{i}}
\end{array}\right.
$$

for every atom $i$ in the system (without lost of generality, we assume $F^{e x t}=0$ ). The superscripts, $\nu$ and $\nu+1$, denote the iteration count within a time window $t \in\left[t_{0}, t_{n}\right]$.

Each atom (or a spatial block consisting of several atoms) in iteration $\nu+1$ is integrated over a time window based on its previous position in time (in the same iteration $\nu+1$ ) and the information about its neighbor positions. As opposed to classical integrators, neighboring positions are taken from the iteration $\nu$. This resembles the Jacobi smoothing for steady state problems. A schematic illustration of 1D WR employing Jacobi splitting is given in Figure 2.

An alternative approach synonymous to the Gauss-Seidel splitting is based on updating internal forces using the information already available from the iteration $\nu+1$. Mathematically, the system is written as

$$
\left\{\begin{array}{l}
m_{i} \ddot{d}_{i}^{\nu+1}=F^{i n t}\left(d_{1}^{\nu+1}, \ldots, d_{i-1}^{\nu+1}, d_{i}^{\nu+1}, d_{i+1}^{\nu}, \ldots, d_{N}^{\nu}\right) \\
d_{i}^{\nu+1}(0)=d_{0_{i}} \\
\dot{d}_{i}^{\nu+1}(0)=v_{0_{i}}
\end{array}\right.
$$

The Gauss-Seidel type approach leads to faster convergence rates obviously at the expense of more limited parallelization. The second variant is known as the Waveform Newton (WN) [26, 27]. The idea is to approximate the internal forces in Eq. (1) as

$$
F^{i n t}=F^{i n t}\left(d^{\nu}\right)+D\left(d^{\nu}\right)\left(d^{\nu}-d^{\nu+1}\right)
$$




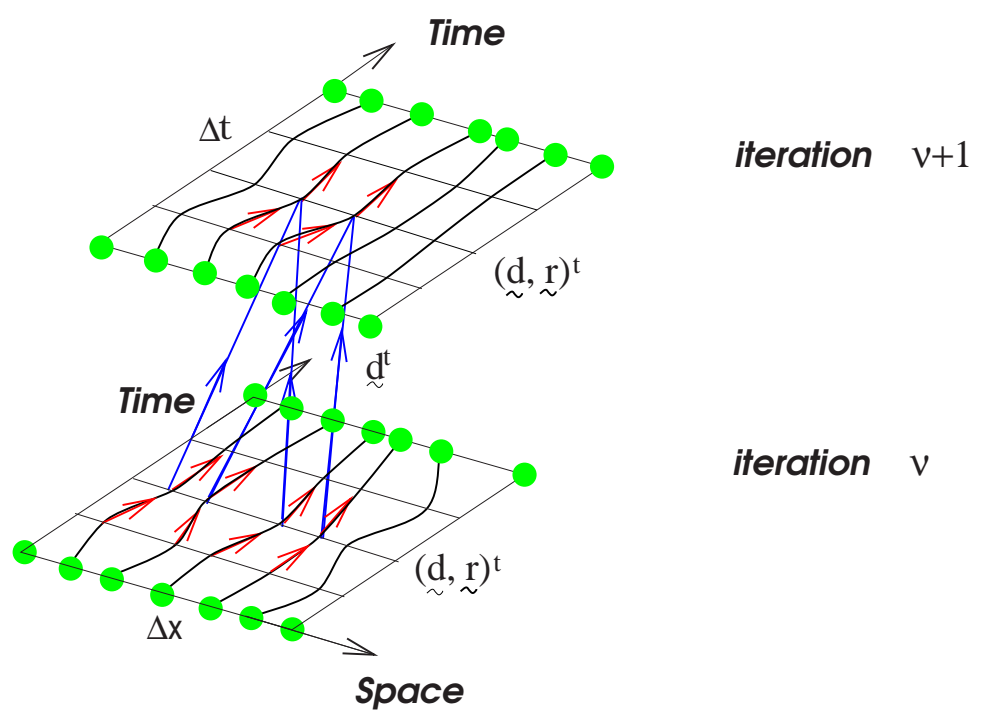

Fig. 2. Jacobi waveform relaxation

where $D\left(d^{\nu}(t)\right)=\operatorname{diag}(H(t))$ is a diagonal of the Hessian matrix obtained from the second derivative of the potential function

$$
H_{i j}(t)=\frac{\partial^{2} \phi(d(t))}{\partial d_{i} \partial d_{j}}
$$

Alternatively, $D$ can be defined as a block diagonal matrix if spatial blocks (see Figure 1) include several atoms. The Hessian matrix may either be computed analytically or 'on the fly' using finite difference approximations.

Substituting the approximation (6) for the internal forces into (1) leads to the following system of ordinary differential equations

$$
\left\{\begin{array}{l}
M \ddot{d}^{\nu+1}+D\left(d^{\nu}\right) d^{\nu+1}=F^{i n t}\left(d^{\nu}\right)+D\left(d^{\nu}\right) d^{\nu} \\
d^{\nu+1}(0)=d_{0} \\
\dot{d}^{\nu+1}(0)=v_{0}
\end{array}\right.
$$

The above system is integrated over the time interval $t \in\left[t_{0}, t_{n}\right]$ using the Newmark predictor-corrector algorithm [28]. Note that if $D$ is diagonal the WR iteration scheme given in Eq. (8) is explicit in nature, even though the proposed space-time multilevel integrator is implicit, allowing for larger time steps. The WR iteration is terminated when the maximum residual in a time window is smaller than a specified tolerance

$$
\max \left\{\left\|r^{\nu+1}(t)\right\|_{2}\right\}=\max \left\{\left\|M d^{\nu+1}-F^{i n t}\left(d_{1}^{\nu+1}, \ldots, d_{i}^{\nu+1}, \ldots, d_{N}^{\nu+1}\right)\right\|\right\} .
$$

or alternatively when

$$
\max \left\{\left\|d^{\nu+1}(t)-d^{\nu}(t)\right\|\right\} \leq \epsilon,
$$


for some small positive constant $\epsilon$. An illustration of the convergence of the WR method to a trajectory of a single atom is shown in Figure 3. Note that

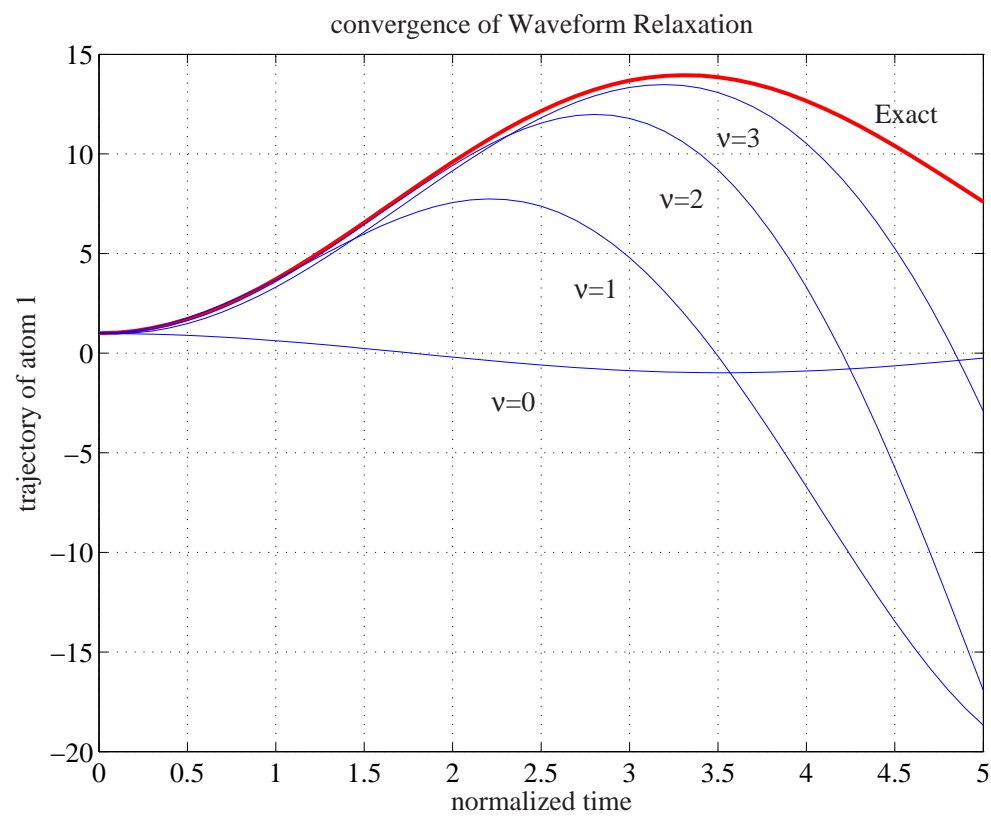

Fig. 3. space-time convergence of waveform relaxation methods

the WR method converges to the entire trajectory. The major drawback of the WR method (both aforementioned versions) is in its slow convergence in case of a strong coupling between spatial blocks and sizable windows [29, 30]. In the proposed variational space-time multilevel approach (presented in Section 3) the WR takes the role of smoother aimed at capturing the high frequency response of atomistic vibrations. To this end we note that the combination of the WR smoothing and the coarse grid correction is not new. The socalled multilevel waveform relaxation (MWR) has been developed in $[31,32]$ in the context of parabolic partial differential equations. However, for highly nonlinear molecular dynamics systems we find this approach either slow to converge or not converging at all.

\section{Variational space-time multilevel method}

We start by a qualitative comparison between the classical spatial multilevel method and the space-time multilevel method presented in this section. For the space-time problem we consider a 1D atomistic chain, which corresponds to the 2D problem in space-time domain, whereas for the spatial structured geometric multilevel method we consider a two-dimensional spatial domain as shown in Figure 4. For the standard multilevel method in 2D the coarse grid correction is provided by the auxiliary coarse grid (black circles and blue 


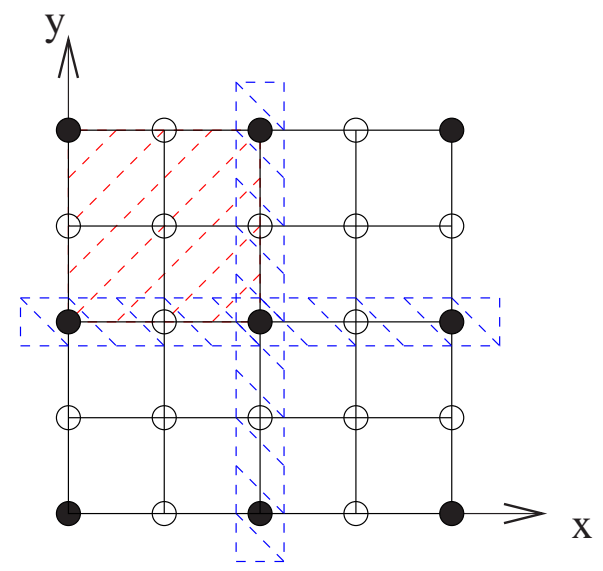

- Coarse

Fine

(a) Standard geometric multilevel

$(1 \mathrm{D}$ space $\times 1 \mathrm{D}$ space $)$

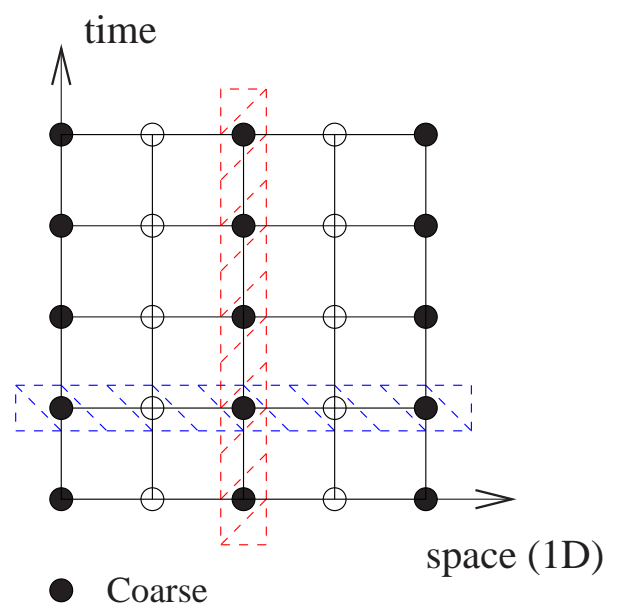

Fine

(b) Space-time multilevel method

$(1 \mathrm{D}$ space $\times$ time $)$

Fig. 4. Standard multilevel in 2D versus space-time multilevel

dashed lines in Figure 4(a)). The smoothing process (red dashed line) is then equivalent to finding the local equilibrium positions of the remaining fine scale degrees-of-freedom (white circles). On the other hand, in the space-time multilevel method, the coarse grid correction corresponds to the solution on a spatial slab (blue dashed lines in Figure 4(b)), whereas smoothing corresponds to the solution of the spatial block on a temporal grid carried out windowby-window to reduce storage (red dashed lines). Note that in the standard multilevel, line smoothing corresponds to solving an algebraic system of equations corresponding to the degrees-of-freedom positioned along the line, while in the space-time variant, line-smoothing is a time integration process.

One of the main challenges in devising an efficient multilevel approach is con- 
structing the coarse scale problem. For continuum systems governed by PDEs, it is possible to construct an auxiliary coarse grid based on PDEs and constitutive equations of the source problem. Thus the coarse grid problem can be directly formulated on the auxiliary coarse model. Employing an analogous approach to molecular equations with force fields computed from the same interatomic potentials for so-called auxiliary representative atoms will result in unrealistic solutions. In other word, the mathematical model for the auxiliary coarse model (often referred to as a coarse grained model) is different than for the original atomistic system.

Developing such an efficient coarse grained model that span considerable length and time scales has been an active research area [33, 34, 35]. An adequate coarse grained model depends on the material system under consideration. For instance, in a polymeric system, the most common coarse grained model is based on the equation of motion of the Langevin type (also known as Brownian dynamics)

$$
M \frac{\partial^{2} R_{i}}{\partial^{2} t}+C \frac{\partial R_{i}}{\partial t}=F_{i}^{s}
$$

where $R_{i}$ is the position of coarse grained particle $\mathrm{i}$ (or blob) M is its mass; $\mathrm{C}$ the equivalent friction between polymer chains; and $F_{i}^{s}$ represents both the Gaussian random forces and the force, which mimic molecular collisions and therefore a thermal reservoir (see for example [33]). Another noteworthy coarse graining approach is the CGMD method [36]. The CGMD constructs coarse grained Hamilton's equations from MD equations under fixed thermodynamics conditions.

In the present manuscript we adopt a different approach by which the coarse grained equations are constructed directly from the fine scale using Hamilton's principle on the subspace of the coarse scale functions. The proposed formulation provides a superior rate of convergence, but is computationally more expensive than evolving the coarse grained model directly since it involves computations of the force fields on the fine scale. Let $e$ be the coarse scale correction aimed at updating the fine scale solution, where $m \ll N$ is the size of the coarse model. The updated fine scale solution at a certain time step is given by $d^{\nu+1} \leftarrow d^{\nu}+Q e$ where $d^{\nu} \in R^{N}$. To find the optimal correction we express the Lagrangian in terms of the correction $Q e$

$$
L(Q e, Q \dot{e})=\frac{1}{2}<M\left(\dot{d}^{\nu}+Q \dot{e}\right),\left(\dot{d}^{\nu}+Q \dot{e}\right)>-\Phi\left(d^{\nu}+Q e\right)
$$

The prolongation $Q$ is assumed to be constant over a certain period of time. By Hamilton's principle $e(t)$ is the minimizer of

$$
S[e(t)]=\int_{t_{1}}^{t_{2}} L(Q e, Q \dot{e}) d t
$$


written as

$$
\frac{\delta S}{\delta e(t)}=0 \quad t_{1}<t<t_{2}
$$

which is equivalent to solving the following Euler-Lagrange equations

$$
\frac{d}{d t}\left(\frac{\partial L}{\partial \dot{e}}\right)-\frac{\partial L}{\partial e}=\frac{\delta S}{\delta e(t)}=0
$$

Substituting the Lagrangian into (15) results in the following coarse grid problem

$$
\left\{\begin{array}{l}
Q^{T} M Q \ddot{e}-Q^{T} F^{i n t}\left(d^{\nu}+Q e\right)=-Q^{T} M \ddot{d}^{\nu} \\
e(0)=0 \\
\dot{e}(0)=0
\end{array}\right.
$$

We will refer to Eq. (16) as the coarse grid correction in space and time. The system (16) can be integrated explicitly or implicitly. For the latter, our experience indicates (see Section 5) that it is sufficient to perform only one or two Newton iterations to reduce the computational cost. Once the error is calculated it is prolongated to the fine scale at each time step within a window. Algorithmic details of the nonlinear space-time multilevel method are summarized in tables corresponding to Algorithms 1,2 and 3. Capital letters indicate matrices; capital letters with subscript $t$ indicate a column in a matrix corresponding to discrete time $t$. $X$ and $A$ denote the space-time atom positions and acceleration matrices, respectively. $M$ and $Q$ are the mass matrix and the coarse-to-fine mapping operator, respectively. We denote $K_{\text {tan }}$ as the tangent stiffness matrix, and $H$ as the Hessian matrix. Lower case letters indicate instantaneous vector quantities: $d, v$ and $a$ are the position, velocity and acceleration vectors, respectively. Indices $h$ and $H$ denote the fine and coarse degrees-of-freedom, respectively. $\Delta t$ is the implicit integration time step. tol is the convergence tolerance. $\beta$ and $\gamma$ are the parameters of the Newmark method. Specifically, we consider the trapezoidal rule with $\beta=\frac{1}{4}$ and $\gamma=\frac{1}{2}$. All Comments are denoted by symbol $\{\%\}$. An illustration of the space-time multilevel method is presented in Figure 5. Arrows depict the information flow.

Remark 1: In the case of Harmonic potentials, i.e. $F^{\text {int }}(d)=-K d$ the coarse grid correction in space and time is consistent with the linear formulations given in [31]

$$
\left\{\begin{array}{l}
Q^{T} M Q \ddot{e}+Q^{T} K Q e=Q^{T} r_{s} \\
e(0)=0 \\
\dot{e}(0)=0
\end{array}\right.
$$

where $r_{s}=-K d_{s}^{\nu+1}-M \ddot{d}_{s}^{\nu+1}$ is the defect computed on the fine scale after smoothing.

Remark 2: The performance of the variational space-time multilevel method can be improved using the windowing technique. By this approach the integration domain is broken into non-overlapping subintervals $\left[0, T_{1}\right],\left[T_{1}, T_{2}\right], \ldots,\left[T_{n-1}, T_{n}\right]$ 


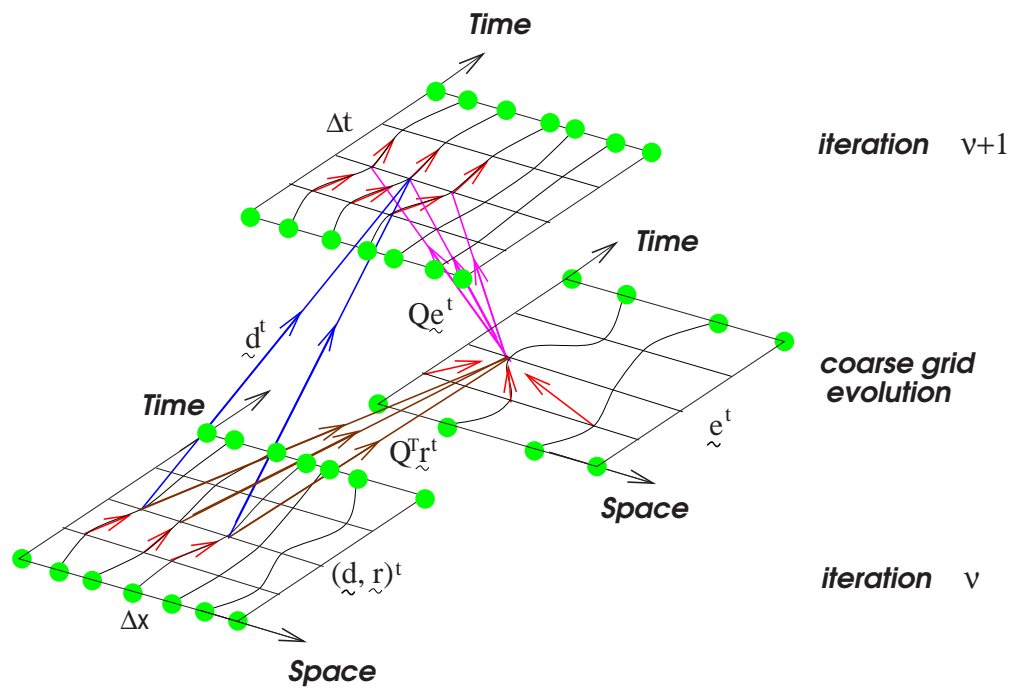

Fig. 5. Space-Time multilevel method
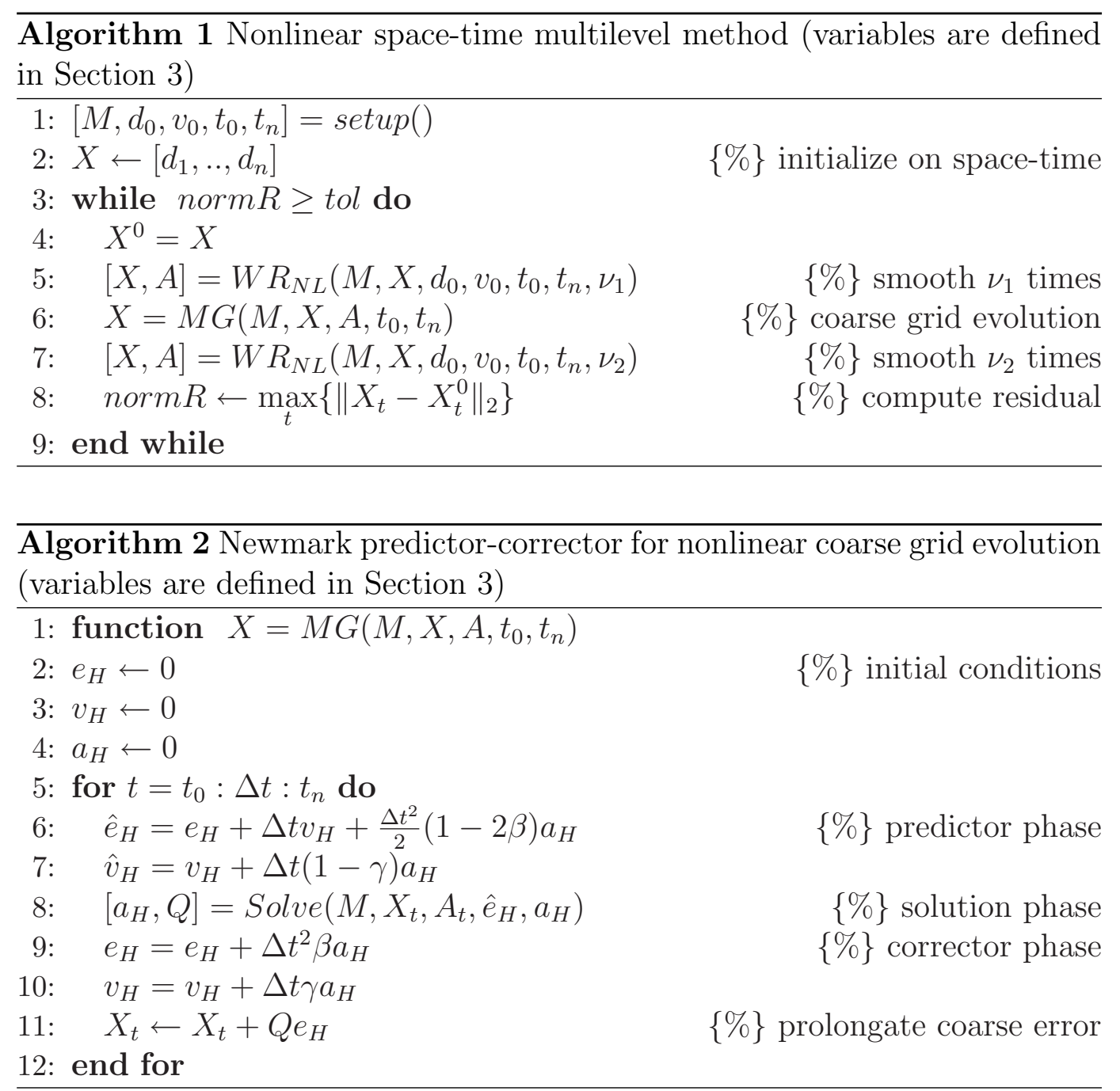


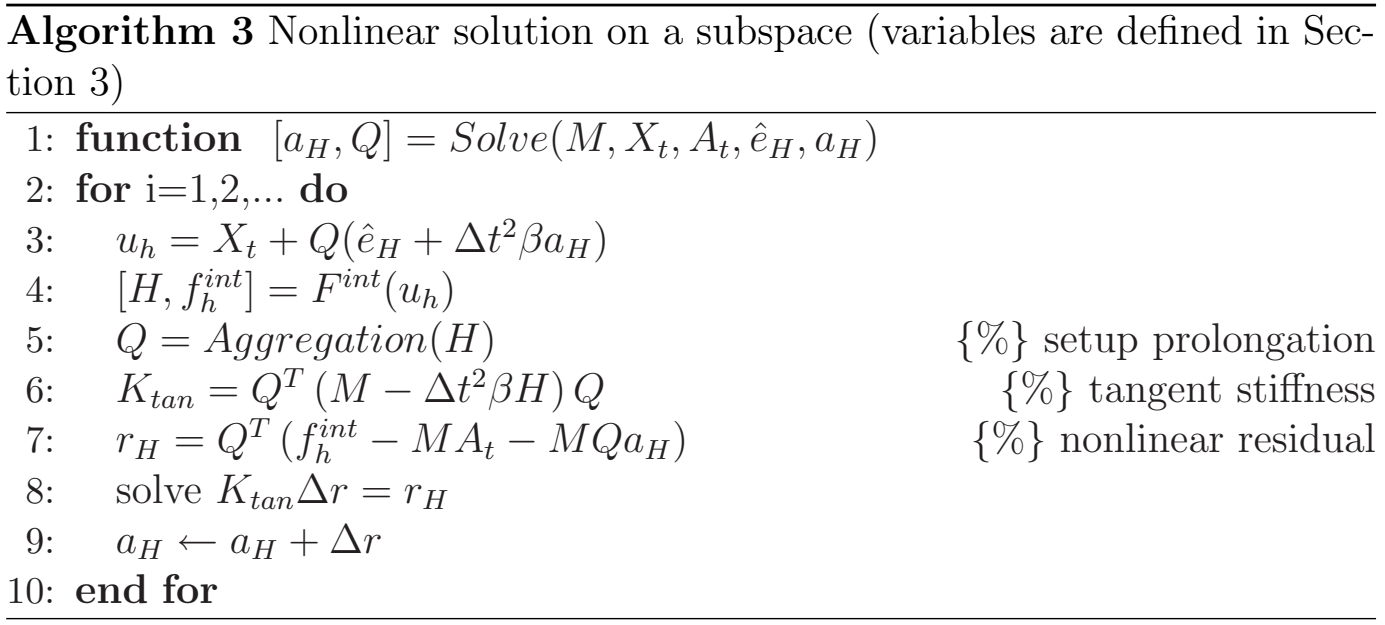

termed windows. The iteration is then performed in each window separately until convergence is reached. The final solution in a certain window is used as an initial condition for the next window. This accelerates the iteration process, reduces the amount of storage and yields better convergence rates [30]. An illustration of such non-overlapping windows is given in Figure 6.

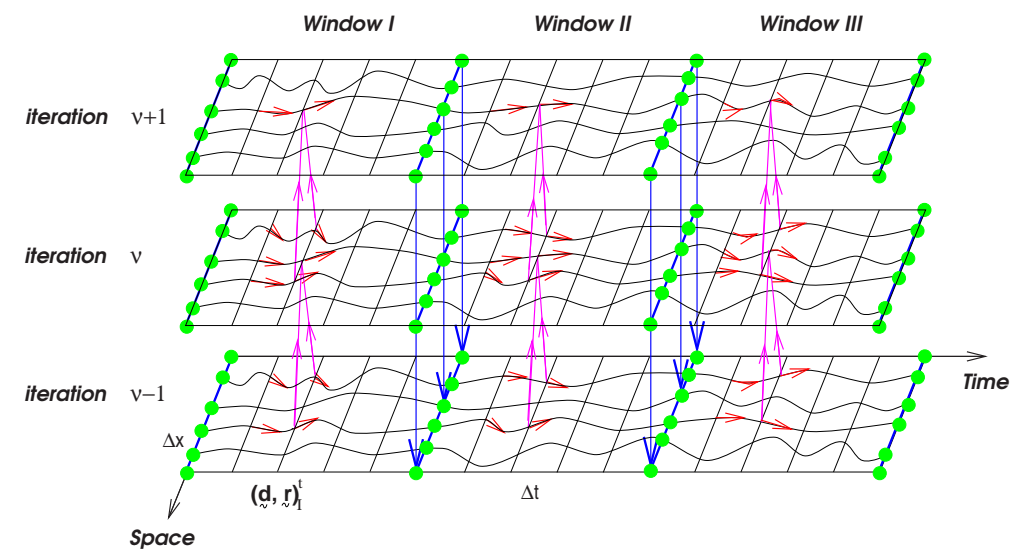

Fig. 6. Non-overlapping windows strategy

Remark 3: Figure 7 illustrates convergence of the space-time multilevel method in a single window. Each line corresponds to the convergence in atom positions (measured in the relative $L_{2}$ norm of the residual $\left(\frac{\left\|r_{i}\right\|_{2}}{\left\|r_{0}\right\|_{2}}\right)$ ) at a certain time step where $\mathrm{i}$ indicates the iteration count. It can be seen that convergence in the initial stages of the integration is faster, or in other words solution in the initial phase converges to a higher tolerance than towards the end of the integration window. This suggests that once the solution in the initial phases has converged, it can be locked (kept fixed) and the iteration could proceed starting with the next time step (within the same window) with the initial values taken from the converged solution. This may reduce the amount of computational cost roughly by a factor of two. 


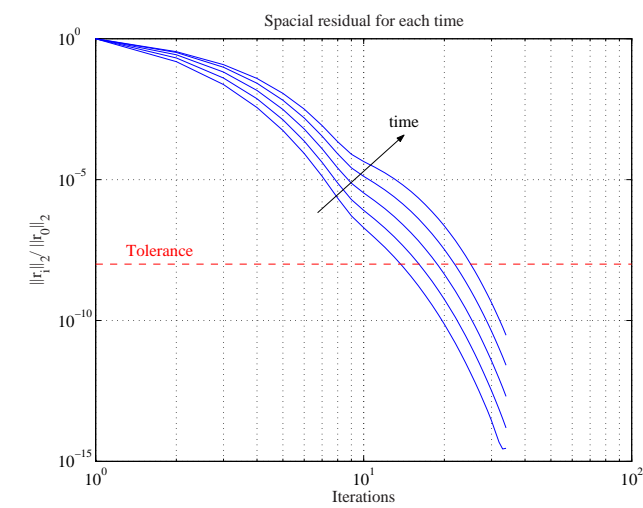

(a) convergence in a window without locking

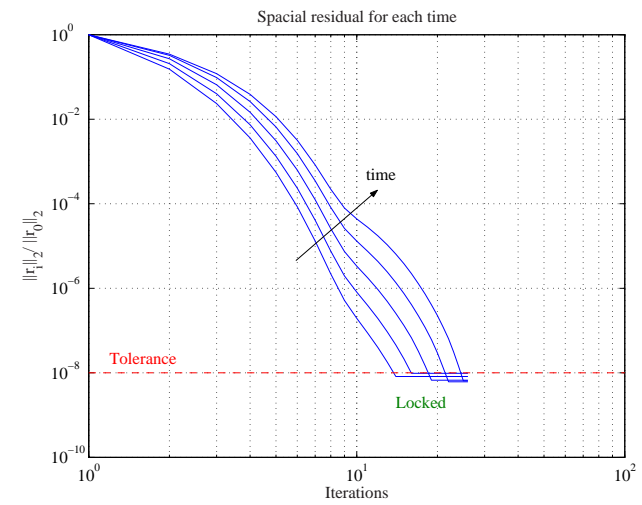

(b) convergence in a window with locking

Fig. 7. Locking strategies to reduce work

\section{Equivalence between the space-time and sequential multilevel methods}

In this Section we show that when the window size coincides with the integration time step and the problem is linear, the proposed space-time multilevel method reduces to the sequential multilevel method applied at each time step [18], for which the convergence studies have been conducted for the model problem [37]. We start with a standard implicit Newmark predictor-corrector integration applied to a system with Harmonic potentials

$$
\left\{\begin{array}{l}
M \ddot{d}+K d=-F^{e x t} \\
d(0)=d_{0} \\
\dot{d}(0)=v_{0}
\end{array}\right.
$$

The atom positions and velocities, $\hat{d}$ and $\hat{v}$, respectively, in the predictor phase are given as

$$
\begin{aligned}
& \hat{d}=d_{0}+\Delta t v_{0}+\frac{\Delta t^{2}}{2}(1-2 \beta) a_{0} \\
& \hat{v}=v_{0}+\Delta t(1-\gamma) a_{0}
\end{aligned}
$$

where the initial acceleration is computed as $\left.a_{0}=M^{-1}\left(-F^{e x t}-K d_{0}\right)\right)$. The resulting accelerations $a$ is

$$
\hat{K} a=-F^{e x t}-K \hat{d}
$$

where

$$
\hat{K}=M+\beta \Delta t^{2} K \text {. }
$$


The corrected values of the displacement and velocity vectors are given by

$$
\begin{aligned}
& d=\hat{d}+\Delta t^{2} \beta a \\
& v=\hat{v}+\Delta t \gamma a
\end{aligned}
$$

In the case of sequential multilevel, one pre-smoothing iteration applied to Eq. (20) gives

$$
\begin{aligned}
a_{s}^{i+1} & =a_{s}^{i}+P_{s}^{-1} r_{s}^{i}=a_{s}^{i}+P_{s}^{-1}\left(-F^{e x t}-K \hat{d}-\hat{K} a_{s}^{i}\right) \\
& =\left(I-P_{s}^{-1} \hat{K}\right) a_{s}^{i}-P_{s}^{-1}\left(F^{e x t}+K \hat{d}\right)
\end{aligned}
$$

where subscript $s$ indicates smoothing, superscript $i$ is the iteration count, $a_{s}$ are the accelerations after smoothing, $r_{s}^{i}$ is the linear system residual and $P_{s}$ is the smoothing preconditioner. For simplicity a Jacobi type smoother is considered, i.e.

$$
P_{s}=M+\beta \Delta t^{2} K_{+} \equiv D
$$

such that $K_{+}=\operatorname{diag}(K)$. The coarse grid correction is given by

$$
Q^{T} \hat{K} Q a=Q^{T} r_{s}^{i+1}
$$

where $a$ is the coarse scale acceleration and $r_{s}^{i+1}=-F^{e x t}-K \hat{d}-\hat{K} a_{s}^{i+1}$ is the residual after smoothing. We now consider the space time variant. The Jacobi WR smoothing with the splitting denoted as $K_{-}=K_{+}-K$ yields

$$
\left\{\begin{array}{l}
M \ddot{d}^{\nu+1}+K_{+} d^{\nu+1}=\left(K_{+}-K\right) d^{\nu}-F^{e x t} \\
d^{\nu+1}(0)=d_{0} \\
\dot{d}^{\nu+1}(0)=v_{0}
\end{array}\right.
$$

Consider one time step integration using the Newmark method. The predictor phase is given by

$$
\begin{aligned}
& \hat{d}^{\nu+1}=\hat{d}=d_{0}+\Delta t v_{0}+\frac{\Delta t^{2}}{2}(1-2 \beta) a_{0} \\
& \hat{v}^{\nu+1}=\hat{v}=v_{0}+\Delta t(1-\gamma) a_{0}
\end{aligned}
$$

The solution phase with the relation (22) becomes

$$
\begin{aligned}
\left(M+\beta \Delta t^{2} K_{+}\right) a^{\nu+1} & =\left(K_{+}-K\right) d^{\nu}-F^{e x t}-K_{+} \hat{d} \\
& =\left(K_{+}-K\right)\left(\hat{d}+\beta \Delta t^{2} a^{\nu}\right)-F^{e x t}-K_{+} \hat{d} \\
& =-F^{e x t}-K \hat{d}+\beta \Delta t^{2} K_{+} a^{\nu}-\beta \Delta t^{2} K a^{\nu} .
\end{aligned}
$$

Addition and subtraction of $M$ from the right hand side leads to

$$
\left(M+\beta \Delta t^{2} K_{+}\right) a^{\nu+1}=-F^{e x t}-K \hat{d}+\left(M+\beta \Delta t^{2} K_{+}-M-\beta \Delta t^{2} K\right) a^{\nu} .
$$


Finally, combining Eqs. (21) and (24) yields

$$
a_{s}^{\nu+1}=\left(I-D^{-1} \hat{K}\right) a^{\nu}-D^{-1}\left(F^{e x t}+K \hat{d}\right),
$$

which is equivalent to Eq. (23) obtained from the smoothing in space. Next, we consider the coarse grid correction in space and time

$$
\left\{\begin{array}{l}
Q^{T} M Q \ddot{e}+Q^{T} K Q e=Q^{T} r_{s} \\
e(0)=0 \\
\dot{e}(0)=0
\end{array}\right.
$$

where $r_{s}=-\left(M d_{s}^{\ddot{\nu+1}}+K d_{s}^{\nu+1}+F^{e x t}\right)$ is the defect computed on the fine scale after smoothing. Once again the solution phase for the coarse scale accelerations $a_{c}$ can be simplified as

$$
\begin{aligned}
Q^{T} M Q a_{c}+\beta \Delta t^{2} Q^{T} K Q a_{c} & =Q^{T}\left(M+\beta \Delta t^{2} K\right) Q a_{c}=-Q^{T}\left(M d_{s}^{\ddot{\nu+1}}+K d_{s}^{\nu+1}+F^{e x t}\right) \\
& =-Q^{T}\left(M a_{s}^{\nu+1}+K d_{s}^{\nu+1}+F^{e x t}\right),
\end{aligned}
$$

and since $a_{s}^{\nu+1}$ and $d_{s}^{\nu+1}$ obtained from the WR smoothing are identical to those obtained from the standard smoothing, it follows that the space-time variant reduces to the sequential multilevel approach. To this end, it is instructive to note that the space-time variant provides a general framework that for the specific case considered in this section coincides with the sequential approach. Convergence estimates for the model problem have been reported in [37].

\section{$5 \quad$ Numerical results}

In this section we conduct performance studies of the space-time multilevel method on a model problem of an atomistic chain and a polymer melt. The MD simulations are performed under conditions of constant N,V,E (the microcanonical ensemble) with consideration of periodic boundary conditions. To asses the accuracy of algorithms we track the following properties: absolute temperature, kinetic energy, configurational (potential) energy, total energy (Hamiltonian) and mean square displacements (self diffusion coefficients). The Newmark predictor-corrector algorithm with parameters $\beta=\frac{1}{4}$ and $\gamma=\frac{1}{2}$ is used for time integration.

\subsection{Atomistic chain with Harmonic potentials}

We first consider an atomistic chain with heterogeneous Harmonic potentials in periodic arrangement with one stiff and one compliant connection; the stiff- 
ness ratio between the two connection is taken as $\frac{k_{s t i f f}}{k_{s o f t}}=100$. We consider the nearest neighbor interactions. The integration time step for the explicit velocity-verlet method is limited by stability considerations. The critical step is obtained by considering how fast a wave travels in the stiffest cell, given by

$$
\Delta t_{c r}=\frac{2}{\sqrt{\rho(A)}},
$$

where $\rho(A)$ is the spectral radius (highest eigenvalue) of $A=M^{-1} K$ [28]. $M$ is a diagonal mass matrix given by $M=m I$ where $m$ is the particle mass; $I$ the identity matrix; and $K$ is the stiffness matrix written as

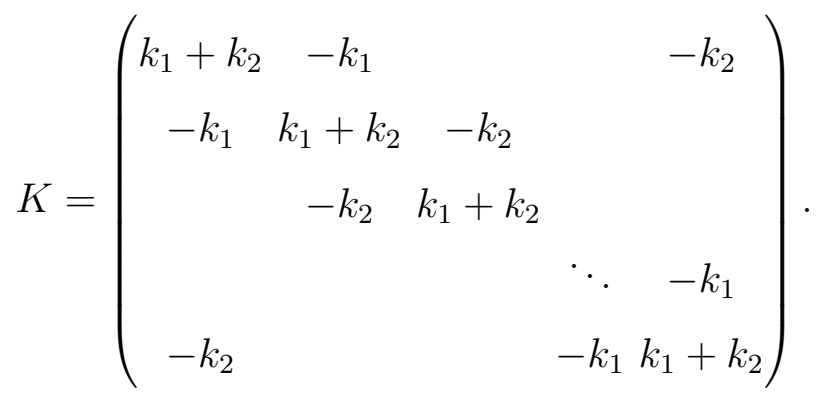

For the multilevel and waveform methods considered, the length of the time step is governed by the accuracy of the coarse fields of interest, selected here both as temperature and self diffusion. The temperature is related to the average kinetic energy of the system and is written as

$$
\text { Temp }=\frac{2}{3 N k_{b}}<E_{k}>
$$

where $N$ is the number of atoms, $k_{b}$ is Boltzmann's constant (here normalized as $k_{b}=\frac{2}{3}$ ) and $\left\langle E_{k}\right\rangle$ is the time average kinetic energy which is a function of atom velocities (see [1] for more details). Figure 8 depicts the relative error in the implicit methods computed as

$$
\operatorname{Er}[\%]=\frac{\left|T e m p_{\text {exp }}-T e m p_{i m p}\right|}{\left|T e m p_{\text {exp }}\right|} \times 100
$$

where Temp $p_{\text {exp }}$ is the temperature obtained by the velocity-verlet method and Temp $p_{i m p}$ is the temperature obtained by the implicit methods for various time steps. Despite the fact that the implicit time step is 40 times larger than that of the explicit scheme the normalized error of the temperature, at steady-state, is less than $0.5 \%$. Consequently, the time step for the space-time multilevel method is chosen as $\Delta t_{i m p}=40 \times \Delta t_{\text {exp }}$. The adequacy of the selected time step is also verified by inspecting the accuracy of the self diffusion coefficient. The diffusion coefficient is obtained from the mean square displacements as

$$
D_{f}=\frac{1}{2 n_{s d}} \lim _{t \rightarrow \infty} \frac{<\left[d\left(t_{0}+t\right)-d\left(t_{0}\right)\right]^{2}>}{t}
$$




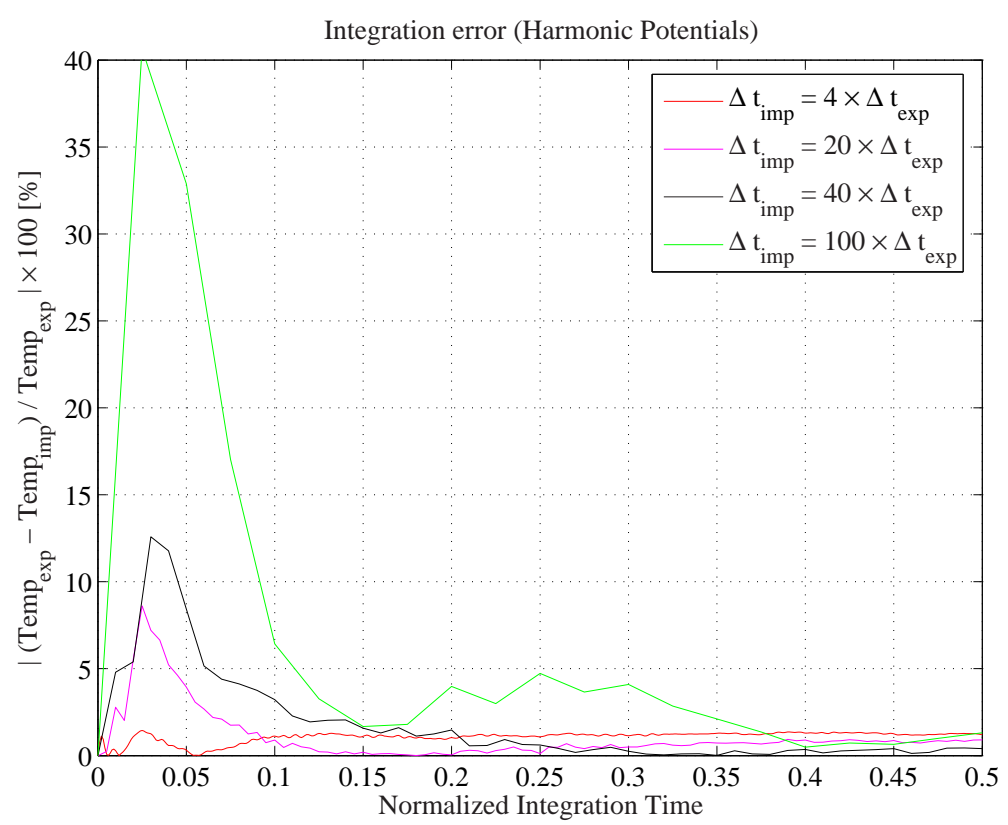

Fig. 8. Error in temperature as a function of the time step length (Harmonic potentials)

where $n_{s d}$ is the number of space dimensions. $t_{0}$ is the time origin for the ensemble time averages. For more details on diffusion coefficients we refer to [1]. Figure 9 illustrates the diffusion behavior (the slope) for both the explicit and implicit methods taken after long times. As before, the implicit time step is taken as $\Delta t_{i m p}=40 \times \Delta t_{\text {exp }}$. An excellent agreement between the methods is observed. Table 1 reports convergence results for the space-time multilevel method compared to the velocity-verlet and waveform relaxation method. The atomic chain consists of $N=1001$ atoms and is integrated over 16 implicit time steps. We use one pre Jacobi smoothing and a coarse grid correction. The coarse grid possess homogenized material properties [19, 20]. Various columns in Table 1 correspond to the type of method, number of windows selected, integration time step (dt), total number of iterations and overall CPU time. All calculations were performed on a single processor machine. It can be seen that the space-time multilevel method has the best performance. To study the windowing strategy, we conduct a series of numerical experiments performed on atomistic chains with various numbers of atoms. The multilevel performance in various windows is illustrated in Figure 10.

\subsection{Polymer melts with Lennard-Jones and Harmonic potentials}

As a second system, we consider a unit cell of a polymer melt as shown in Figure 11. Various colors correspond to different chains where every chain consists of 16 atoms. Lennard-Jones (LJ) potentials are used to model the interaction 


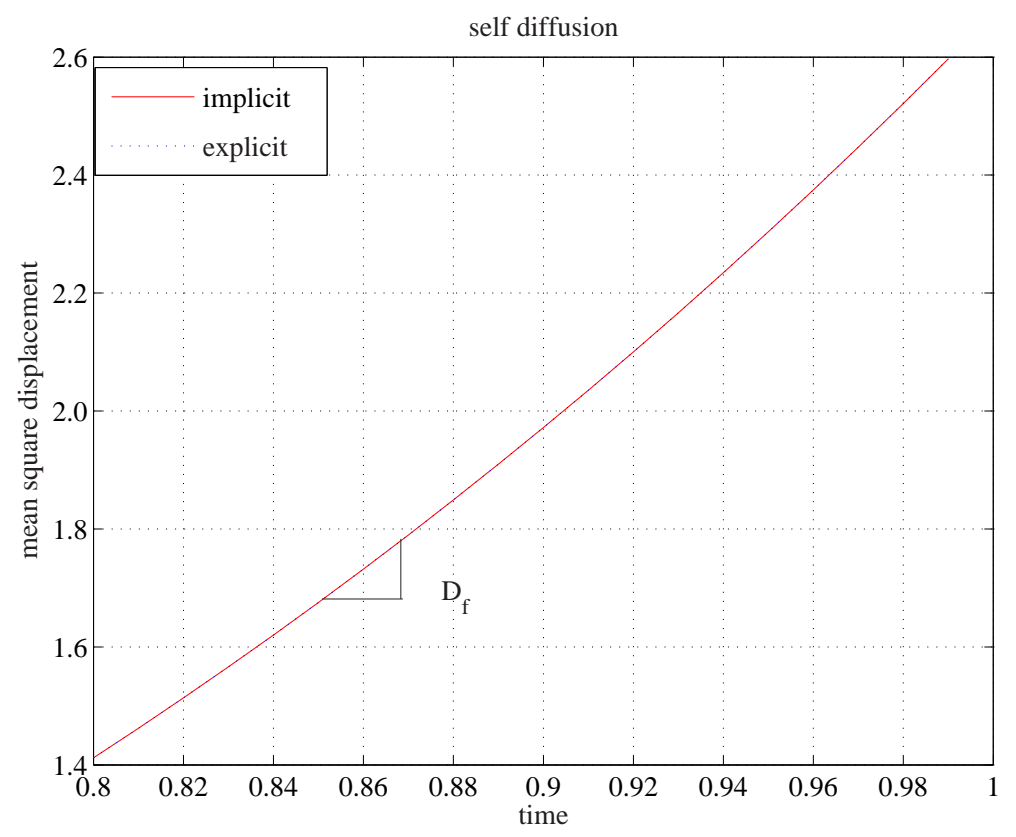

Fig. 9. Diffusion coefficient for both explicit and implicit methods. $\Delta t_{\text {imp }}=40 \times \Delta t_{\text {exp }}$

Table 1

CPU time and iteration summary for a $1 \mathrm{D}$ system with Harmonic potentials

\begin{tabular}{|l||c|c|c|c|}
\hline Method & Windows & $\mathrm{dt}$ & iterations & $C P U$ time [sec] \\
\hline \hline MG-space-time & 1 & $4 \times 10^{-2}$ & 11 & 27.39 \\
MG-space-time & 2 & $4 \times 10^{-2}$ & 14 & 22.68 \\
MG-space-time & 4 & $4 \times 10^{-2}$ & 24 & 22.14 \\
MG-space-time & 8 & $4 \times 10^{-2}$ & 36 & 20.45 \\
MG-space-time & 16 & $4 \times 10^{-2}$ & 64 & 22.07 \\
WR & 1 & $4 \times 10^{-2}$ & 256 & 226.76 \\
WR & 2 & $4 \times 10^{-2}$ & 347 & 226.18 \\
WR & 4 & $4 \times 10^{-2}$ & 352 & 153.43 \\
WR & 8 & $4 \times 10^{-2}$ & 386 & 113.70 \\
WR & 16 & $4 \times 10^{-2}$ & 588 & 101.95 \\
velocity-verlet & - & $1 \times 10^{-3}$ & - & 83.15 \\
\hline
\end{tabular}

between all pairwise atoms in the system (all atoms including atoms in different chains), and Harmonic potentials are added along the polymer axis (only 


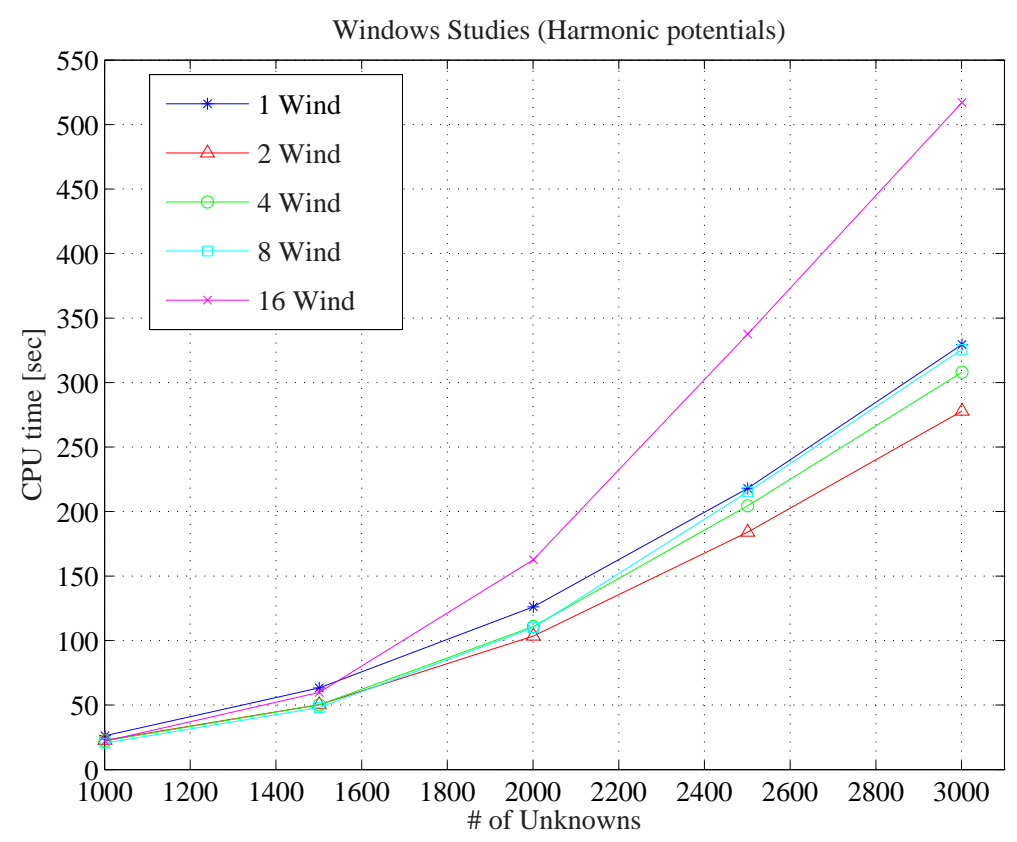

Fig. 10. Windowing strategies in multilevel (Harmonic potentials)

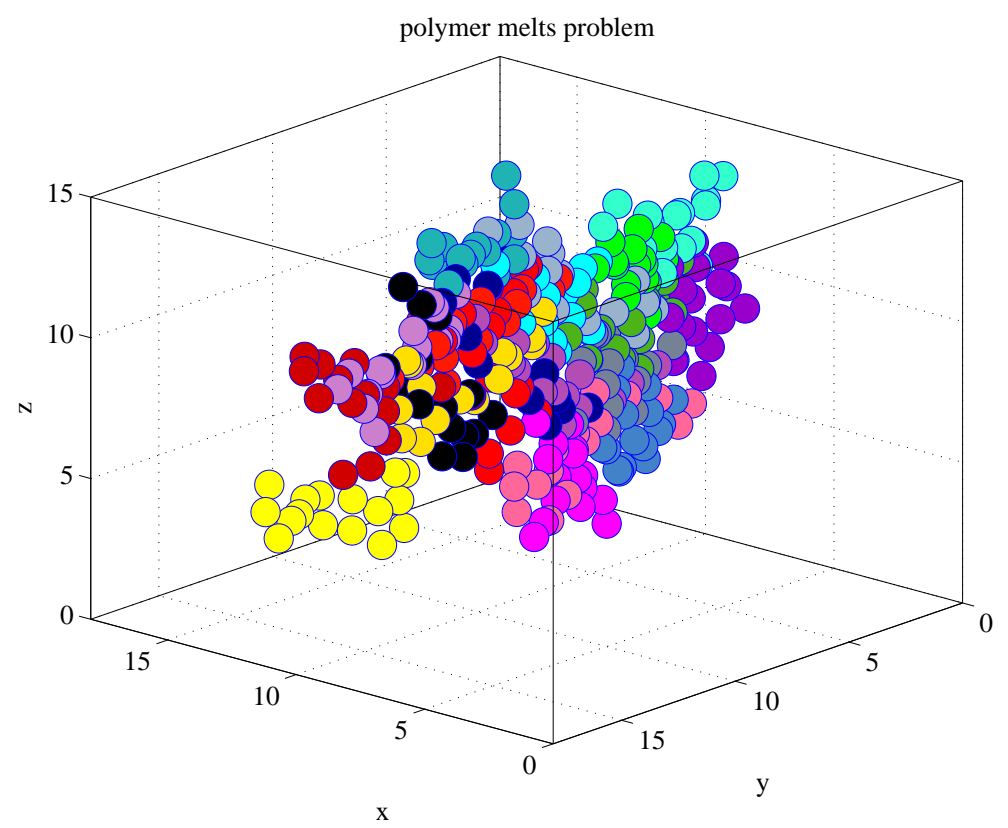

Fig. 11. 20 polymer chains consisting of 16 atoms per chain

within a chain). Mathematically we have

$$
\Phi=\sum_{i=1}^{N} \sum_{j>1}^{N-1} \Phi_{L J}\left(r_{i j}\right)+\frac{1}{2} \sum_{b o n d s} k_{c}^{\text {bond }}\left(r_{c}-r_{0}\right)^{2} .
$$

The LJ potential describes the energy of interaction between two atoms as a function of the distance between their centers $r_{i j}$. The standard pairwise LJ 
potential is given by

$$
\Phi_{L J}\left(r_{i j}\right)=4 \epsilon\left[\left(\frac{\sigma}{r_{i j}}\right)^{12}-\left(\frac{\sigma}{r_{i j}}\right)^{6}\right]
$$

where $r_{i j}=\sqrt{\left(r_{x, i}-r_{x, j}\right)^{2}+\left(r_{y, i}-r_{y, j}\right)^{2}+\left(r_{z, i}-r_{z, j}\right)^{2}} ; \epsilon$ is the well-depth of the interaction and $\sigma$ is the collision diameter. The total potential energy is given as a sum over all pairs

$$
\Phi=\frac{1}{2} \sum_{i=1}^{N} \sum_{\substack{j=1 \\ i \neq j}}^{N} \Phi_{L J}\left(r_{i j}\right)=\sum_{i=1}^{N} \sum_{j>1}^{N-1} \Phi_{L J}\left(r_{i j}\right) .
$$

In our simulations we use normalized units with $\epsilon=\sigma=1$ and heterogeneous Harmonic potentials to model the chains. That is, different bond stretching constants $k_{c}^{b o n d}$ are used for different chains. Specifically,

$$
k_{c}^{\text {bond }}= \begin{cases}1 & c=1,3, \ldots \\ 10 & c=2,4, \ldots\end{cases}
$$

and $r_{0}=1$. This heterogeneity creates a range of time scales, and thus the explicit velocity-verlet algorithm, due to its stability requirement, is limited by the fast vibrating components. As before, we control the accuracy in temperature given by Eq. (33), and diffusion coefficient calculated in Eq. (35). Figure 12 shows the normalized error in temperature for 20 polymer chains that consist of 16 atoms per chain (see Figure 11). We use the explicit method with $\Delta t_{\text {exp }}=1 \times 10^{-3}$ as the reference solution. The allowable error in temperature is selected to be $1.5 \%$. This corresponds to the implicit time step that is 20 times larger than the explicit time step. The system is integrated over 16 implicit steps. The diagonal terms of the Hessian matrix in Eqs. (7)(8) are computed analytically. The comparison of the diffusion coefficient for $\Delta t_{i m p}=20 \times \Delta t_{\text {exp }}$ is given in Figure 13. The system is integrated over 40000 explicit time. It can be seen that the multilevel method predicts the diffusion coefficient with only $1 \%$ of error compared to the explicit method. The details of the algorithm are given below. We use one presmoothing of Jacobi waveform Newton (WN). The iteration is terminated when $\left\|d^{\nu+1}-d^{\nu}\right\|_{2} \leq 10^{-4}$ for all times. The prolongation operator in the space-time multilevel method is computed based on the aggregation approach [38]. Specifically, we form a local Hessian matrix for every aggregate $H^{a g g}(t)$ (see Eq. (7)). Each aggregate corresponds to a single polymer chain. The size of the matrix is $n_{d c} \times n_{d c}$, where $n_{d c}=48$ is the number of degrees-of-freedom associated with each polymer chain. The Hessian matrix for a single aggregate is formed from LJ and harmonic potentials as

$$
H^{a g g}=H^{L J}+H^{\text {harmonic }}=\frac{\partial^{2} \phi_{L J}}{\partial d_{i} \partial d_{j}}+\frac{\partial^{2} \phi_{\text {harmonic }}}{\partial d_{i} \partial d_{j}} .
$$




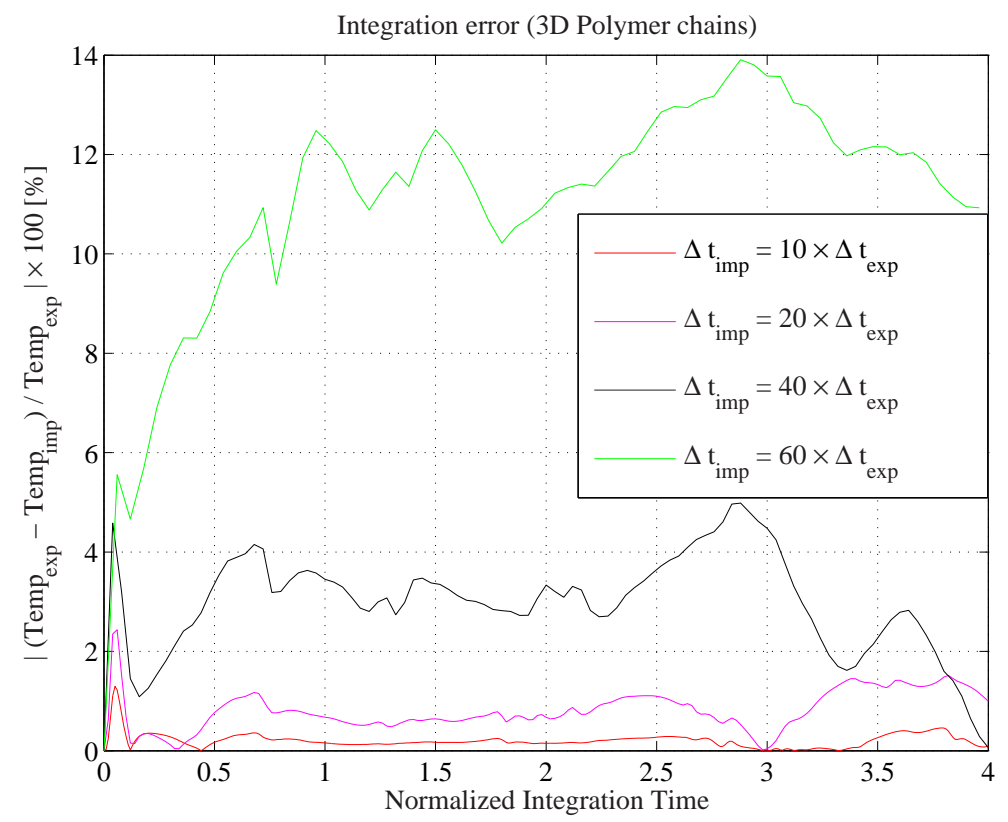

Fig. 12. Error in temperature as a function of the time step (3D polymer chains)

Table 2

CPU time and iteration summary for 8 polymer chains (16 atoms per chain) with mixed Lennard Jones and Harmonic (heterogeneous) potentials

\begin{tabular}{|l||c|c|c|c|c|c|c|}
\hline Method & Winds & $\mathrm{dt}$ & iter. & Nonlin. iter. & Func. eval. & Eigen [sec] & $C P U[\mathrm{sec}]$ \\
\hline \hline MG-space-time & 4 & $20 \times 10^{-3}$ & 21 & 60 & 121 & 13.98 & 428.016 \\
MG-space-time & 8 & $20 \times 10^{-3}$ & 25 & 43 & 87 & 10.01 & 303.484 \\
MG-space-time & 16 & $20 \times 10^{-3}$ & 33 & 33 & 67 & 5.92 & 225.891 \\
WN & 4 & $20 \times 10^{-3}$ & 46 & - & 151 & - & 491.141 \\
WN & 8 & $20 \times 10^{-3}$ & 70 & - & 125 & - & 415.063 \\
WN & 16 & $20 \times 10^{-3}$ & 106 & - & 107 & - & 357.672 \\
velocity-verlet & 1 & $1 \times 10^{-3}$ & - & - & 321 & - & 1049.72 \\
Implicit Newmark & 1 & $20 \times 10^{-3}$ & - & 112 & 113 & - & 380.156 \\
\hline
\end{tabular}

$H^{L J}$ is a dense matrix, as all atoms are assumed to interact, while $H^{\text {harmonic }}$ is block diagonal matrix with $6 \times 6$ blocks. Based on the aggregation approach, the prolongation operator $Q$ is constructed by solving the following eigenproblem for the lowest modes of every polymer chain

$$
H^{a g g} v_{i}=\lambda_{i} v_{i} \quad i=1, . ., n_{m}
$$




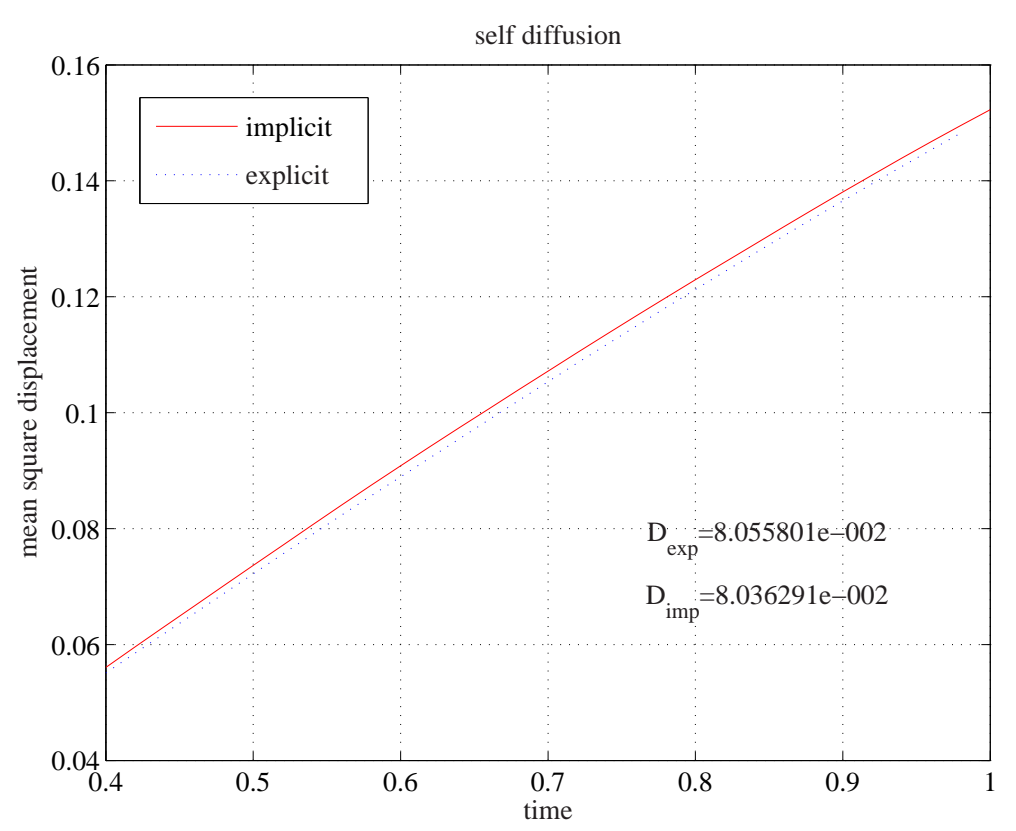

Fig. 13. Diffusion coefficient for both explicit and implicit methods. $\Delta t_{i m p}=20 \times \Delta t_{\text {exp }}$

where $n_{m}$ is the number of modes. In the present case we selected $n_{m}=32$. We next define the subspace

$$
V_{j}=\operatorname{span}\left\{v_{1}, \ldots, v_{n_{m}}\right\} \quad j=1, . ., n_{c}
$$

where $n_{c}$ is the number of polymer chains. The prolongation operator is then formulated as

$$
Q=\left(\begin{array}{llll}
V_{1} & & & \\
& V_{2} & & \\
& & \ddots & \\
& & & V_{n_{c}}
\end{array}\right)_{\left(n_{d c} \times n_{c}\right) \times\left(n_{m} \times n_{c}\right)} .
$$

The CPU time required to construct the prolongation is reported in the Table 2. For the problem considered, it is found to be negligible compared to the overall CPU time.

For the numerical experiments considered the space-time multilevel method outperformed the explicit and WR methods. The best performance on a single processor machine is obtained when the window size is equal to the size of the time step.

Next, we study the performance of the space-time multilevel method as the number of polymer chains increase. Figures 14(a) and 14(b) illustrates the $\mathrm{CPU}$ times on a single processor machine for the explicit and the space-time multilevel method as the number of polymer chains increases (each chain consists of 16 atoms) in one integration time step. The explicit method requires 21 force computations while the multilevel only needs 5 . It can be seen that 


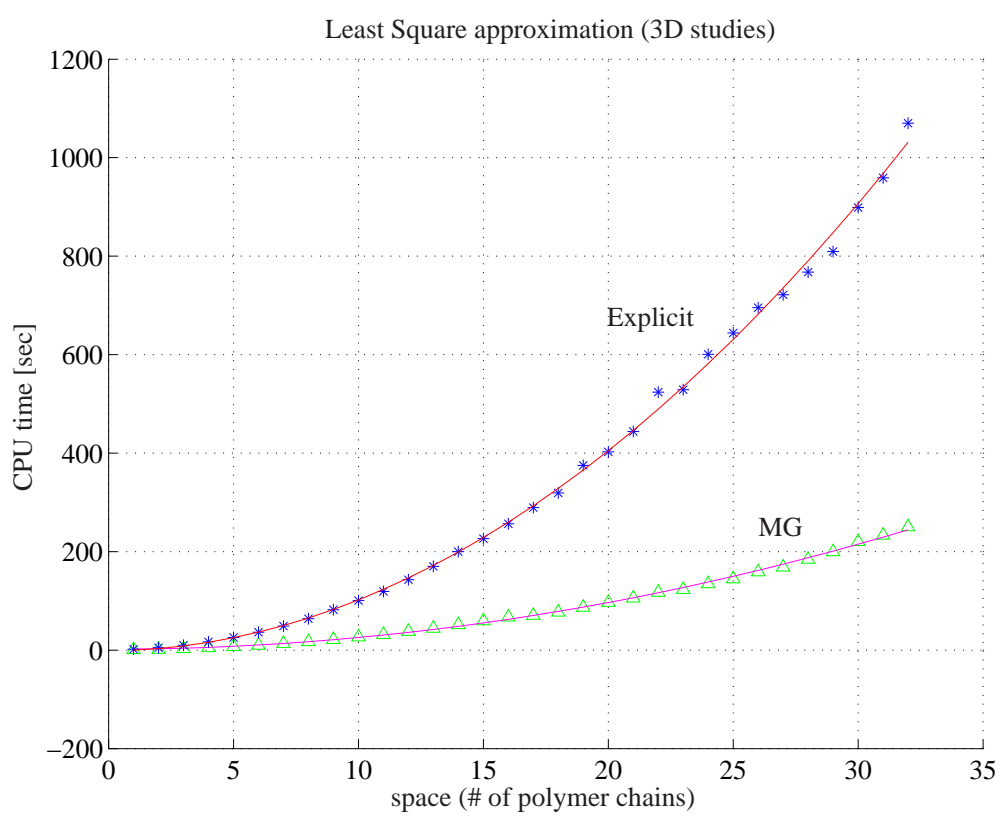

(a) CPU times after one time step

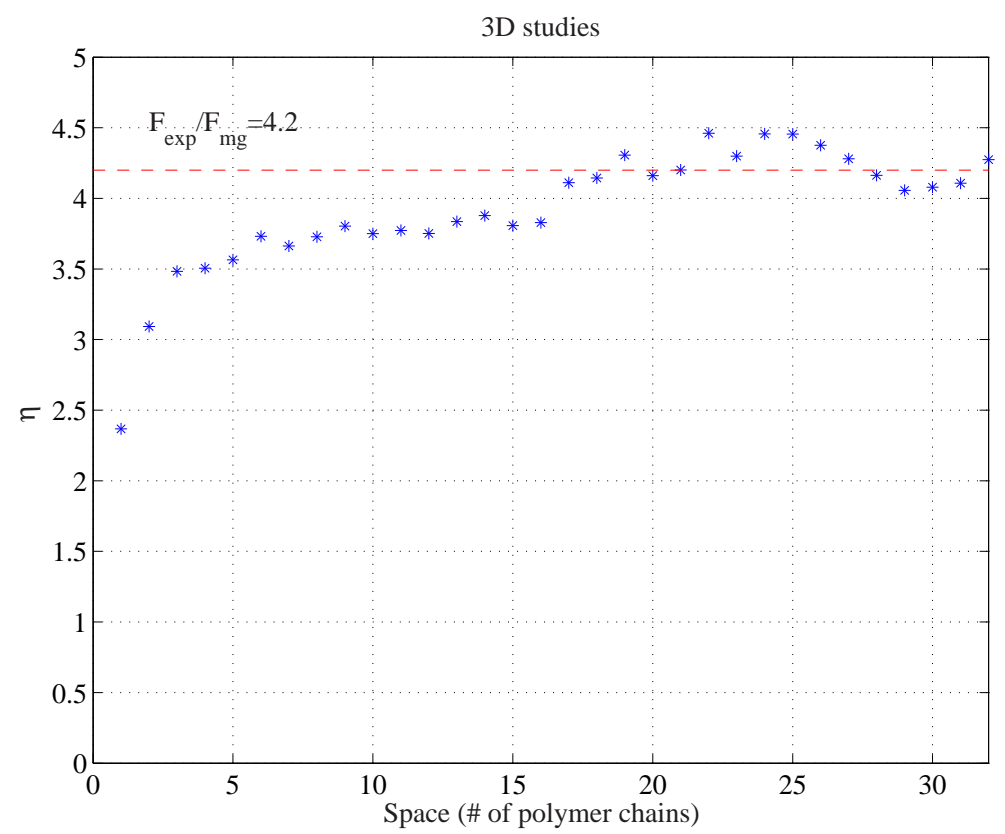

(b) CPU time ratio between the explicit and multilevel methods

Fig. 14. CPU time studies

the speed-up factor has been found to be around 4 independent of problem size. We note that the use of a neighbor list approach [1] would have reduced the computational complexity of both methods. The space-time multilevel method would have resulted in sparser Hessian matrix, more efficient WR and coarse grid correction in addition to cost savings in force field calculations which would have been the main cost savings for explicit methods. Finally, we 
study the stability properties of the space-time multilevel method by considering a long time interval. We integrate the system over 40000 explicit time steps (2000 implicit steps). Figure 15 depicts fluctuations in the configurational (potential) energy $U$ and the total energy $E_{\text {tot }}$ (Hamiltonian). It can be seen that the proposed multilevel approach is stable as the Hamiltonian fluctuates around an average value. This average value differs by only $\% 0.18$ from the value obtained by the explicit method.
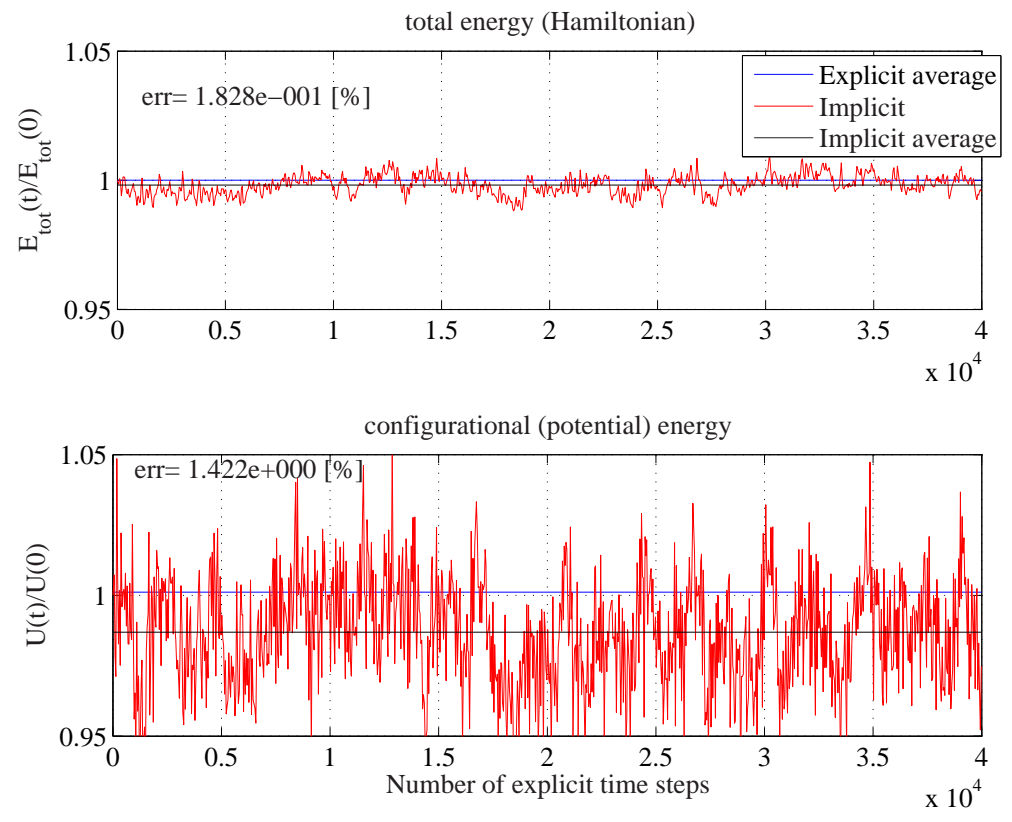

Fig. 15. Stability of multilevel method on a long time interval

\subsection{Parallel implementation for Harmonic potentials}

In this section we consider implementation of the explicit and space-time multilevel methods on a single processor, two-processor, four-processor and 16processor machines for the polymer melt unit cell described in the previous section. We use the ML package developed at Sandia for the parallel implementation [39]. Table 3 and Figure 16 demonstrate the time parallelization effect on the speed-up factor over the explicit method. The number of polymer chains in a unit cell is indicated in the first column and performance of explicit and space-time multilevel methods is compared on several processors. We use the same material parameters as Section 5.1. We apply one pre-smoothing of WR-Jacobi iteration with no coarsening in space, i.e., the prolongation operator coincides with the identity matrix. The resulting coarse scale problem is solved approximately using a single iteration with Jacobi preconditioner. Numerical integration is carried out in a single window with eight implicit steps. The results clearly show that as the number of processors increases, the speed- 
Table 3

Parallel CPU times for polymer unit cell integrated over one window (8 implicit steps)

\begin{tabular}{|l||c|c||c|c||c|c||c|c|}
\hline \multirow{2}{*}{$\begin{array}{l}\text { \# of polymer } \\
\text { chains }\end{array}$} & \multicolumn{2}{c||}{ P1 } & \multicolumn{2}{c||}{ P2 } & \multicolumn{2}{c||}{ P4 } & \multicolumn{2}{c|}{ P16 } \\
\hline \hline 340 & MG & Exp & MG & Exp & MG & Exp & MG & Exp \\
\hline 1365 & 0.51 & 2.32 & 0.31 & 1.45 & 0.17 & 0.81 & 0.03 & 0.21 \\
\hline 5460 & 2.30 & 10.84 & 1.47 & 7.06 & 0.71 & 3.44 & 0.16 & 0.99 \\
\hline
\end{tabular}

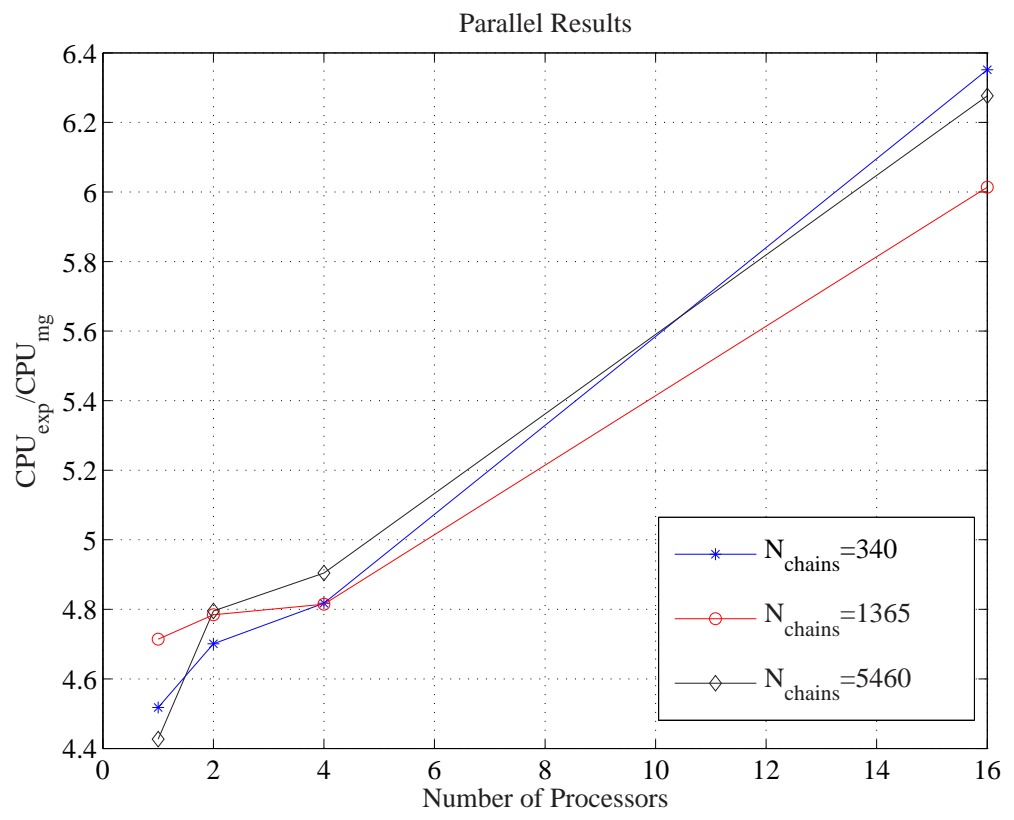

Fig. 16. Multilevel parallel results (polymer unit cell)

up factor between the MG and velocity-verlet methods increases. The main reason for the increase in the speed-up factor is due to processors communication effect. In the case of standard explicit or implicit methods processors are communicating after every time step. However, in the MG and WR case the communication between processors only takes place at the end of each window. This results in superior parallel performance as has been suggested in $[31]$.

\section{CONCLUSIONS}

A space-time multilevel method is developed to accelerate time integration of molecular dynamics equations. The method consists of the waveform relaxation smoothing aimed at capturing the oscillatory response of the atomistic 
vibrations and a coarse grained model evolution intended to resolve the smooth features of the atomistic media. The formulation of the coarse grained model is based on the variational approach derived from the Hamilton's principle. The time integration is performed in windows using the Newmark predictorcorrector method. The integration scheme is implicit and thus larger time steps governed by accuracy rather than by stability requirements may be taken. Numerical examples show significant time savings compared to standard explicit integrators. A parallel version of the space-time multilevel method has been developed for harmonic potentials. Significant speed-ups over the standard explicit method have been observed primarily due to reduced communication time between the processors. Future work will include (i) consideration of coarse grids based on the Brownian dynamics and other coarse-grained models and (ii) parallel implementation for various interatomic potentials.

\section{Acknowledgements}

The financial supports of National Science Foundation under grants CMS0310596, 0303902, 0408359 and Sandia National Laboratory under contract DE-ACD4-94AL85000 are gratefully acknowledged. In particular, the authors wish to thank Dr. Ray Tuminaro from Sandia National Laboratory for his constructive suggestions

\section{References}

[1] J. M. Haile. Molecular dynamics simulation elementary methods. John Wiley \& Sons, INC., 1992.

[2] L. Verlet. Computer "experiments" on classical fluids. i. thermodynamical properties of lennard-jones molecules. Physical Review, 159(1):98-103, 1967.

[3] W. C. Swope, H. C. Anderson, P. H Berens, and K. R. Wilson. A computer simulation method for the calculation of equilibrium constants for the formation of physical clusters of molecules: Application to small water clusters. The Journal of Chemical Physics, 76(1):637-649, 1982.

[4] C. W. Gear. Numerical initial value problems in ordinary differential equations, chapter 9. Prentice-Hall, 1971.

[5] C. Bennemann, W. Paul, K. Binder, and B. Dnweg. Molecular-dynamics simulations of the thermal glass transition in polymer melts: $\alpha$-relaxation behavior. Physical Review E, 57(1):843-851, 1998.

[6] D. C. Rapaport. The art of molecular dynamics simulations. Cambridge university press, 1995.

[7] H. C. Anderson. Rattle: a velocity version of the shake algorithm for mole- 
cular dynamics calculations. Journal of Computational Physics, 52:24-34, 1983.

[8] M. Tuckerman, B. J. Berne, and G. J. Martyna. Reversible multiple time scale molecular dynamics. The Journal of Chemical Physics, 97(3):19902001, 1992.

[9] B. Garcia-Archilla, J. M. Sanz-Serna, and R. D. Skeel. The mollified impulse method for oscillatory differential equations. SIAM Journal on Scientific Computing, 20:930-963, 1998.

[10] E. Hairer, C. Lubich, and G. Wanner. Geometric Numerical Integration. Structure-Preserving Algorithms for Ordinary Differential Equations. Springer, 2002.

[11] D. Barash, L. Yang, X. Qian, and T. Schlick. Inherent speedup limitations in multiple time step/particle mesh ewald algorithms. Journal of Computational Chemistry, 24:77-88, 2003.

[12] B. J. Leimkuhler, S. Reich, and R. D. Skeel. Integration methods for molecular dynamics. IMA Volumes in Mathematics and its Applications, 82:161-186, 1997.

[13] D. Janezic and B. Orel. Implicit Runge-Kutta method for molecular dynamics integration. Journal of Chemical Information and Computer Sciences, 33:252-257, 1993.

[14] C. Sagui and T. Darden. Multigrid methods for classical molecular dynamics simulations of biomolecules. The Journal of Chemical Physics, 114(15):6578-6591, 2001.

[15] R. D. Skeel, I. Tezcan, and D. J.Hardy. Multiple grid methods for classical molecular dynamics. Journal of Computational Chemistry, 23(6):673684, 2002.

[16] G. Sutmann and B. Steffen. A particle-particle particle-multigrid method for long-range interactions in molecular simulations. Computer Physics Communications, 169(1-3):343-346, 2005.

[17] S. Goedecker, F. Lanon, and T. Deutsch. Linear scaling relaxation of the atomic positions in nanostructures. Physical Review B, 64(161102):1-4, 2001.

[18] J. Fish and W. Chen. Discrete-to-continuum bridging based on multigrid principles. Computer Methods in Applied Mechanics and Engineering, 193:1693-1711, 2004.

[19] J. Fish and V. Belsky. Multigrid method for periodic heterogeneous media part 1: Convergence studies for one-dimensional case. Computer Methods in Applied Mechanics and Engineering, 126:1-16, 1995.

[20] J. Fish and V. Belsky. Multi-grid method for periodic heterogeneous media part 2: Multiscale modeling and quality control in multidimensional case. Computer Methods in Applied Mechanics and Engineering, 126:1738, 1995.

[21] W. Hackbusch. Multi-grid methods and applications. Springer, 1985.

[22] E. Lelarasmee, A. E. Ruehli, and A. L. Sangiovanni-Vincentelli. The waveform relaxation method for time-domain analysis of large scale inte- 
grated circuits. IEEE transactions on computer-aided design of integrated circuits and systems, CAD-1(3):131-145, 1982.

[23] K. Burrage. Parallel and sequential methods for ordinary differential equations. Oxford University Press, 1995.

[24] S. Ta"san and H. Zhang. Fourier-laplace analysis of multigrid waveform relaxation method for hyperbolic equations. Technical Report ICASE 96-53, NASA, 1996.

[25] B. Leimkuhler. Timestep acceleration of waveform realxation. SIAM Journal on Numerical Analysis, 35(1):31-50, 1998.

[26] R. Saleh and J. White. Accelerating relaxation algorithms for circuit simulation using waveform-newton and step-size refinement. IEEE Transactions on computer-aided design, 9(9):951-958, 1990.

[27] Andrew Lumsdaine and Mark W. Reichelt. Waveform iterative techniques for device transient simulation on parallel machines. In Sixth SIAM Conference on Parallel Processing for Scientific Computing, Norfolk, VA, 1993.

[28] T. J. R. Hughes. The finite element method. Linear static and dynamic finite element analysis. Prentice-Hall, 1987.

[29] U. Miekkala and O. Nevanlinna. Convergence of dynamic iteration methods for initial value problems. SIAM Journal on Scientific and Statistical Computing, 8(4):459-482, 1987.

[30] E. Giladi and H. B. Keller. Space time domain decomposition for parabolic problems. Numerische Mathematik, 93(2):279-313, 2002.

[31] S. Vandewalle. The parallel solution of parabolic partiall differential equations by multigrid waveform relaxation methods. $\mathrm{PhD}$ thesis, Katholike Universiteit Leuven, 1992.

[32] G. Horton and S. Vandewalle. A space-time multigrid method for parabolic partial differential equations. SIAM Journal on Scientific and Statistical Computing, 16(4):848-864, 1995.

[33] J. T. Padding and W. J. Briels. Coarse-grained molecular dynamics simulations of polymer melts in transient and steady shear flow. The Journal of Chemical Physics, 118(22):10276-10286, 2003.

[34] J. T. Padding and W. J. Briels. Time and length scales of polymer melts studied by coarse-grained molecular dynamics simulations. The Journal of Chemical Physics, 117(2):925-943, 2002.

[35] R. L. C. Akkermans and W. J. Briels. Coarse-grained dynamics of one chain in a polymer melt. The Journal of Chemical Physics, 113(15):64096422, 2000.

[36] R. E. Rudd and J. Q. Broughton. Coarse-grained molecular dynamics and the atomic limit of finite elements. Physical Review B, 58(10):R5893R5896, 1998.

[37] J. Fish and W. Chen. Rve-based multilevel method for periodic heterogeneous media with strong scale mixing. Journal of Engineering Mathematics, 46:87-106, 2003.

[38] J. Fish and V. Belsky. Generalized Aggregation Multilevel solver. Interna- 
tional Journal for Numerical Methods in Engineering, 40(23):4341-4361, 1997.

[39] C. Tong and R. S. Tuminaro. ML 2.0 smoothed aggregation user's guide. Technical Report SAND2001-8028, Sandia National Laboratories, 2000. 